BNL-73615 -2005-CP

\title{
Transverse Spin at PHENIX and Future Plans
}

\author{
Y. Makdisi for the PHENIX Collaboration
}

Presented at $16^{\text {th }}$ International SPIN Physics Symposium

Trieste, Italy, October 10-16, 2004

January 2005

Collider-Accelerator Department

Brookhaven National Laboratory

P.O. Box 5000

Upton, NY 11973-5000

www.bnl.gov

Managed by

Brookhaven Science Associates, LLC

for the United States Department of Energy under

Contract No. DE-AC02-98CH10886 


\section{DISCLAIMER}

This report was prepared as an account of work sponsored by an agency of the United States Government. Neither the United States Government nor any agency thereof, nor any of their employees, nor any of their contractors, subcontractors, or their employees, makes any warranty, express or implied, or assumes any legal liability or responsibility for the accuracy, completeness, or any third party's use or the results of such use of any information, apparatus, product, or process disclosed, or represents that its use would not infringe privately owned rights. Reference herein to any specific commercial product, process, or service by trade name, trademark, manufacturer, or otherwise, does not necessarily constitute or imply its endorsement, recommendation, or favoring by the United States Government or any agency thereof or its contractors or subcontractors. The views and opinions of authors expressed herein do not necessarily state or reflect those of the United States Government or any agency thereof. 


\title{
TRANSVERSE SPIN AT PHENIX AND FUTURE PLANS
}

\author{
Y. MAKDIST FOR THE PHENIX COLLABORATION \\ Brookhaven National Laboratory, Bldg. 911 \\ Upton, NY 11973, USA \\ E-Mail: makdisi@bnl.gov
}

\begin{abstract}
The PHENIX experiment took data with transversely polarized proton beams in 2001-2002 and measured the transverse single spin asymmetries in inclusive neutral pion and non-identified charge hadrons at midrapidity and $\sqrt{s}=200 \mathrm{GeV}$. The data near $\mathrm{X}_{F} \sim 0$ cover a transverse momentum range from 0.5 to $5.0 \mathrm{GeV} / \mathrm{c}$. The observed asymmetries are consistent with zero with good statistical accuracy. This paper presents the current work in light of earlier measurements at lower energies in this kinematic region and the future plans of the PHENIX detector.
\end{abstract}

\section{Introduction}

Large transverse single spin asymmetries in $\mathrm{p} \uparrow \mathrm{p}$ collisions have been observed in a number of experiments at energies ranging from $\sqrt{s}=5-10 \mathrm{GeV}$. Asymmetries approaching $30 \%$ were observed in inclusive pion production at large $x_{F}{ }^{1,2}$. In the kinematic region at midrapidity at high $x_{T}$ and $p_{T}$ up to $2 \mathrm{GeV} / \mathrm{c}$, large asymmetries were also observed in inclusive $\pi^{0}$ and $\pi^{+}$production but not in $\pi^{-}$production ${ }^{3,4,5}$. From the low energy data one could surmise that the asymmetries tend to grow and reach a maximum near the kinematic limits. At higher energies $\sqrt{s}$ of 20 and $200 \mathrm{GeV}$ where pQCD is considered applicable, asymmetries at large $x_{F}$ persisted ${ }^{6,7}$, however, the asymmetry in $\pi^{0}$ production at midrapidity was found to be zero up to $p_{T}$ of $4 \mathrm{GeV} / \mathrm{c}^{8}$.

The data presented here were collected by the PHENIX detector in 2001-2002 with RHIC running with transversely polarized proton beams at $\sqrt{s}=200 \mathrm{GeV}$. At these energies the measured unpolarized cross sections at midrapidity were found to agree well with the NLO pQCD calculations ${ }^{13}$ providing confidence that perturbative calculations are applicable to the polarized data, Figure 1. 


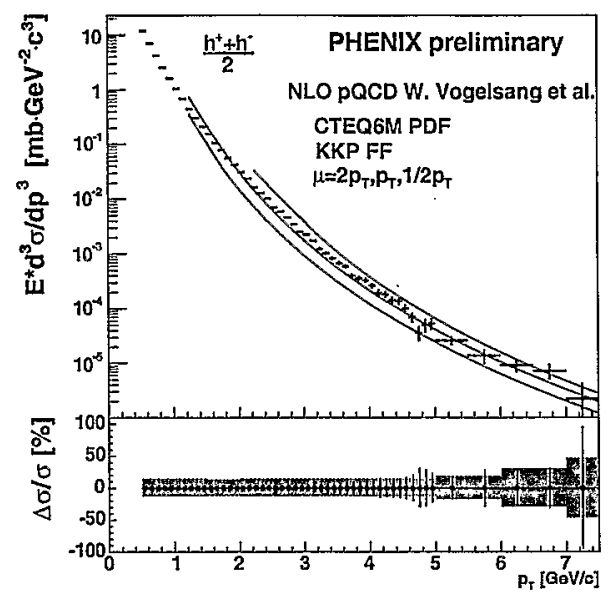

Figure 1. Invariant cross section vs. $p_{T}$. The curves respresent NLO pQCD calculations.

\section{The experimental set up and data analysis}

The RHIC beams had 56 bunches each, an average intensity of $5 \cdot 10^{10}$ per bunch, and bunch polarization +-+- and ++-- in each beam respectively. A total of $0.15 \mathrm{pb}^{-1}$ were accumulated. The average beam polarization was $15 \%$ as measured by the p-Carbon CNI polarimeters with an overall scale uncertainty of $35 \%$ most of which is attributed to the lack of analyzing power of the physics process ${ }^{9}$. The relative luminosity between bunch collisions was determined for each fill using two sets of $2 \pi$ azimuthally symmetric Beam Beam Counters (BBC) located at $\pm 1.44 \mathrm{~m}$ from the interaction point. This was confirmed from a later run by a comparison with the Zero Degree Calorimeters at $\pm 18 \mathrm{~m}$ that are sensitive to very forward neutrons. The accuracy was determined to about $2.5 \cdot 10^{-4}$.

The collision trigger utilized a coincidence between the BBC's which accounts for approximately $50 \%$ of the proton-proton inelastic cross section at $200 \mathrm{GeV}$ and provides minimal bias to a vertical beam polarization. With a vertex position resolution of $\approx 2 \mathrm{~cm}$ the trigger accepted events within $75 \mathrm{~cm}$. The analyzed sample had a vertex cut of $\pm 30 \mathrm{~cm}$. In order to enhance the neutral pion sample, a photon energy threshold of $0.8 \mathrm{GeV}$ was added in coincidence. Charge particle reconstruction was carried out using the drift and pad chambers which cover $|\eta|<0.35$ and $\Delta \phi=90^{\circ}$. Conversion electrons were reduced to less than $1 \%$ by requiring no hits in 


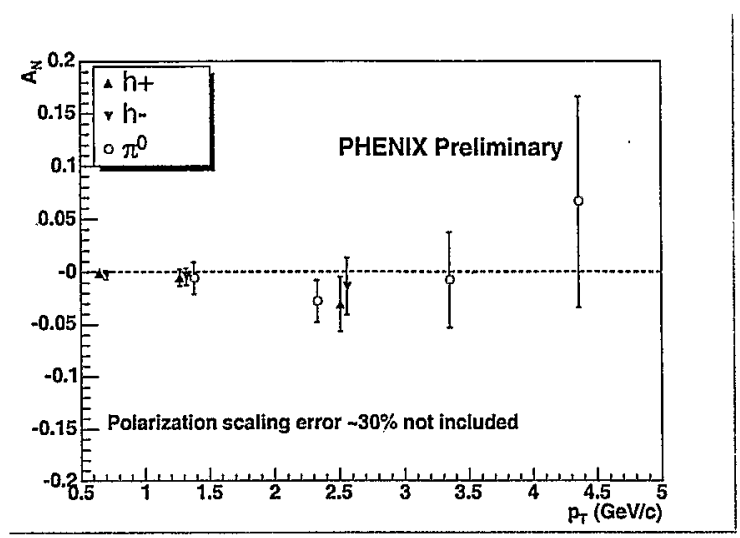

Figure 2. Single spin asymmetries in inclusive $\pi^{0}$ and unidentified hadron production.

the RICH counter with a pion threshold of $4.7 \mathrm{GeV} / \mathrm{c}$. Decays from short lived particles could not be removed and accounted for less than $5 \%$ at the highest momenta.

The two photon effective mass was reconstructed using the EMcalorimeters with trigger efficiencies ranging from $24 \%$ to $78 \%$ and the $\pi^{0}$ peak widths ranged from 13.2 to $10.6 \mathrm{MeV} / \mathrm{c}^{2}$ from low to high $p_{T}$. The yield was extracted at each transverse momentum within a mass cut of $0.12-0.16 \mathrm{GeV} / \mathrm{c}^{2}$. The asymmetry in the background was calculated using $50 \mathrm{MeV} / \mathrm{c}^{2}$ bins around the $\pi^{0}$ mass.

The asymmetries were calculated using the luminosity formula for each fill and then averaged over the whole run. With both beams polarized, single spin analyses were carried out independently using one polarized beam while averaging over the other. To assess the systematics, a bunch shuffing technique was also employed in which the spin direction of each bunch was assigned randomly assuring the same relative luminosities. The results indicate systematic uncertainties smaller than the statistical ones.

\section{The results}

The asymmetries in inclusive production at midrapidity and very low $\mathrm{x}_{T}$ in both neutral and unidentifed charged hadrons are consistent with zero to within a few percent (figure 2). The data represent the first measurements at this energy and kinematic region. It is interesting to note that at low transverse momenta below $p_{T}$ of $3 \mathrm{GeV} / \mathrm{c}$ gluon-gluon partonic interactions dominate. These results could be indicative of a low gluon contribution to 
transverse asymmetries. The data are consistent with the results seen by E704 ${ }^{8}$. These should be contrasted with the large asymmetries observed at $\mathrm{STAR}^{7}$ and more recently BRAHMS (unpublished) in inclusive pion production at forward $\mathrm{x}_{F}$ where valence quark interactions are likely to dominate.

Recently several theoretical models ${ }^{10,11,12}$ have been advanced to explain these asymmetries including higher-twist contributions, modifications to the parton distribution functions (Sivers effect), modifications to the fragmentation functions (Collins effect). These tend to explain the large $\mathrm{x}_{F}$ asymmetries but they have yet to delve into the midrapidity domain.

\section{Future plans}

Since these data were taken, significant progress has been attained in luminosity as well as the polarization such that it is reasonable to assume accumulating more than $1 \mathrm{pb}^{-1}$ per week of running at polarization exceeding $40 \%$ (Run 5 in 2005). With $3 \mathrm{pb}^{-1}$ PHENIX could extend the reach to $p_{T} \approx 10 \mathrm{GeV} / \mathrm{c}$ with better than $5 \%$ statistical accuracy well into the regime of gluon-quark interactions. The instailation of an aerogel counter will extend the particle identification range from the current $3 \mathrm{GeV}$ using the TOF to about $9 \mathrm{GeV}$. The charged hadron trigger at higher momenta can be augmented by the RICH.

In addition, PHENIX is planning to explore the Sivers effect using leading back to back hadrons. Further out with the planned upgrade of a barrel Si vertex detector, back to back jets can be studied as well as exploration of the Collins effect using $\pi \pi$ interference which requires higher luminosities.

\section{References}

1. W.H. Dragoset et al., Phys. Rev. D18 (1978) 3939-3954.

2. C.E. Allgower et al., Phys.Rev.D65 (2002) 092008.

3. J. Antille et al., Phys.Lett.B94 (1980) 523.

4. S. Saroff et al. Phys. Rev. Lett. 64 (1990) 995.

5. V.D. Apokin et al., Phys. Lett. B243 (1990) 461-464.

6. D.L. Adams et al., Phys. Lett. B261 (1991) 201.

7. J. Adams et al., Phys. Rev. Lett. 92 (2004) 171801.

8. D.L. Adams et al., Phys. Lett. B264 (1991) 462.

9. O. Jinnouchi et al., 15th Int.Spin Phys.Symp.AIP Conf.Proc. 675 (2003) 817.

10. D.W. Sivers, Phys. Rev. D41 (1990) 83 and Phys. Rev. D43 (1991), 261.

11. J.C. Collins, Nucl. Phys. B396 (1933) 161.

12. J.W. Qui and G. Sterman, Phys. Rev. D59 (1999) 014004.

13. W. Vogelsang et al. Phys. Rev. D67 (2003) 054005. 